\title{
Enhancing the quality and transparency of reporting
}

\section{The Nature journals' recently updated reporting requirements for life sciences aim to enhance the quality and transparency of methodological and experimental reporting.}

The Nature journals have a well-established commitment to improving the reporting standards of the papers we publish. These efforts led to the introduction in May 2013 of the reporting checklist (Nat. Cell Biol. 15, 443; 2013 and Nature 496, 398; 2013), a document prompting authors to provide information on methodological and analytical aspects of their submitted papers. The reporting checklist was developed following discussions with researchers about the challenges in enhancing research reproducibility, including a series of workshops organized by the National Institutes of Health, and also by drawing on the cumulative experience of the editors of the Nature journals on reporting requirements. Nature Cell Biology authors and referees are by now very familiar with the journal's original customized reporting checklist in which authors were asked to disclose elements of methodology, experimental design, data analysis and presentation, including descriptive statistics, to assist editors and referees in their assessment of the technical quality and robustness of the data. Although the reporting checklist was not published, it served as a valuable tool for authors, editors and referees in ensuring that all pertinent information was also included in the manuscript itself.

We have recently taken an additional step in enhancing the transparency and clarity of reporting in our papers by updating the reporting guidelines for life-sciences research papers published in the Nature journals (http://go.nature.com/2tdbbs1 and Nature 546, 8; 2017). The original reporting checklist has been replaced by a reporting summary document, which captures a similar set of methodological, reagent and data analysis information that are common across multiple life-sciences research areas, and which may contribute to a lack of reproducibility when not appropriately reported. This updated document is not only available to editors and referees during the peer-review process, but is also published as part of the manuscript files for all papers accepted for publication after 31 May 2017.

The multidisciplinary nature of research papers and wealth of methodologies they may include means that this reporting summary is not exhaustive. To capture key technical aspects of specific experimental approaches it is accompanied by methodspecific supplemental reporting summaries that are also published with the paper. These are currently available for chromatin immunoprecipitation sequencing, flow cytometry and magnetic resonance imaging data, but will later expand to include more techniques. We have also introduced an editorial policy checklist in order to help authors ensure that specific requirements of reporting and compliance with editorial policy are met.

As in the case of the original reporting checklist, these initiatives do not intend to enforce a specific set of standards, but rather aspire to capture the key elements of how experiments were designed, conducted and analysed, so that referees are able to assess the technical quality and rigour of the data, and others are able to replicate and build on the findings following publication.

The Nature journals have long championed greater transparency in experimental reporting and data presentation. For many years Nature Cell Biology has mandated the provision of unprocessed scans of key blots and gels shown in the manuscript, and recommended the inclusion of the raw data underlying graphical representations, in the form of a supplementary table that is accessible directly from the relevant figure legends. We have also long encouraged authors to favour graphical representations that display the complete set of data. Aiming to enhance the clarity of data presentation and statistical descriptions in our papers, we are now requiring that all data points are shown especially when sample sizes are low, and that rather than bar graphs, approaches are adopted that present the full spread of the data, for example box plots or violin plots.

Adding to these efforts, we have expanded the well-established policy of the Nature journals regarding mandatory data deposition for specific large-scale data types, to include proteomics data. Information on how these and other data that support the findings of the paper may be accessed is provided in the data availability statements that have been mandated for all research papers published in the Nature journals since 2016 (Nat. Cell Biol. 19, 259; 2017). We have also recently introduced a reporting table for cryo-electron microscopy, much in the vein of existing ones for nuclear magnetic resonance imaging and $\mathrm{X}$-ray crystallography experiments.

To further aid the reproducibility of results, we continue to encourage sharing of detailed experimental procedures through online resources such as the Protocol Exchange, a free-to-use, open resource into which authors can upload experimental protocols and then link these to the Nature Cell Biology paper in which they were used. Nature Cell Biology requires protocol deposition for all Technical Reports and all papers in which capturing the detailed step-by-step nuances of an experimental approach is deemed critical.

The irreproducibility of scientific results is a complex and multifaceted problem - tackling it will require the concerted efforts of research institutes, scientific organizations, funders and publishers. Investing more heavily in rigorous training of students and scientists from the earlier stages of their careers will be essential. The commitment of the scientific community to instil the core principles of robust experimental design and the value of replication to budding scientists is also key. With the initiatives outlined here, we continue to contribute to these efforts and we hope that our authors will appreciate the significance of raising reporting standards to enhance the robustness and reproducibility of the research we publish. 